\title{
In Defense of Mathematical Inferentialism
}

\begin{abstract}
I defend a new position in philosophy of mathematics that I call mathematical inferentialism. It holds that a mathematical sentence can perform the function of facilitating deductive inferences from some concrete sentences to other concrete sentences, that a mathematical sentence is true if and only if all of its concrete consequences are true, that the abstract world does not exist, and that we acquire mathematical knowledge by confirming concrete sentences. Mathematical inferentialism has several advantages over mathematical realism and fictionalism.
\end{abstract}

\section{Keywords}

Concrete Adequacy, Mathematical Fictionalism, Mathematical Inferentialism, Mathematical Realism

Park, Seungbae (2017). "In Defense of Mathematical Inferentialism", Analysis and Metaphysics 16: 70-83.

https://www.addletonacademicpublishers.com/contents-am/986-volume-16-2017/3033-indefense-of-mathematical-inferentialism

I am grateful to the three anonymous reviewers of this journal for their meticulous and insightful comments.

Seungbae Park

Ulsan National Institute of Science and Technology

Republic of Korea

nature@unist.ac.kr

\section{Introduction}

Mathematical realism asserts that mathematical objects, such as numbers, triangles, and functions, exist in the abstract world, and that a mathematical sentence is true if and only if the abstract world is as it says it is. This view is defended by such philosophers as Willard V. O. Quine (1948; 1980), Hilary Putnam (1971), Michael Resnik (1997), Mark Colyvan (2001), and Alan Baker $(2005 ; 2009 ; 2012)$. In contrast, mathematical fictionalism asserts that mathematical sentences purport to be about the abstract world, but the abstract world does not exist, so they are all false. This view is defended by such philosophers as Mark Balaguer (1996; 1998; 2001; 2009), Gideon Rosen (2001), and Mary Leng (2005a; 2005b; 2010).

This paper defends a new position that I call mathematical inferentialism. The discussion proceeds as follows. In Section 2, I explicate mathematical inferentialism and display its virtues. I then reply to possible objections to this position. Next, I list the disadvantages of mathematical realism in Section 3 and the disadvantages of mathematical fictionalism in Section 4. It will become clear that mathematical inferentialism secures the objectivity of mathematics and explains the successful use of mathematics in empirical science without postulating the existence of the abstract world. (From now on, I drop the qualifier 'mathematical' in front of the name of a philosophical view.)

\section{Inferentialism}

\subsection{Content}


Instrumentalism in philosophy of science is the view that a scientific theory is not a description of unobservables but an instrument for organizing our thoughts about observables. The instrument is neither true nor false. It is only useful or useless, depending on whether or not it makes predictions that turn out to be true. Andreas Osiander (1498-1552) was an instrumentalist about the Copernican theory (Kuhn, 1957: 187). George Berkeley (16851753) was an instrumentalist about Newtonian mechanics (Downing, 2013). The insight of these instrumentalists is that a scientific theory can help us to make inferences about observables, i.e., it exhibits the inferential relationship between observational sentences.

As Pierre Duhem (1905) observes, an observational sentence cannot be derived from a scientific theory alone, and a scientific theory needs to be supplied with auxiliary assumptions to entail an observational consequence. What if all of its observational consequences are true? According to Bas C. van Fraassen (1980), such a theory is empirically adequate. On his account, a scientific theory is a description of both observables and unobservables. So it is true or false, depending on whether it correctly represents them. But the principle of economy dictates that we believe only that it is empirically adequate (van Fraassen, 1985: 294).

In my view, instrumentalism in philosophy of science is potentially applicable to philosophy of mathematics. A mathematical sentence can perform the function of helping us to make deductive inferences from some concrete sentences to other concrete sentences, sentences that are rendered true or false by the concrete world. Consider the following three concrete sentences:

(i) John gave me an apple.

(ii) Jane gave me an apple.

(iii) Therefore, I had two apples.

Assume that I had no apples to begin with, and that John and Jane were my only sources for apples. The mathematical sentence, ' $1+1=2$,' tells us that (iii) necessarily follows from (i) and (ii), thereby facilitating the deductive inference from (i) and (ii) to (iii). To generalize, a mathematical sentence can perform the function of helping us deductively organize our thoughts about the concrete world. This functional thesis about a mathematical sentence is the key constituent of what I call inferentialism.

Inferentialism proposes that a mathematical sentence is concretely adequate if and only if all of its concrete consequences are true, and that it is concretely inadequate if and only if a limited number of concrete consequences are true. A concrete consequence is not derivable from a mathematical sentence alone. A mathematical sentence needs to be supplied with concrete auxiliaries. For example, ' $1+1=2$ ' needs to be conjoined with (i) and (ii) to entail (iii). ' $1+1=2$ ' is concretely adequate because it entails only true concrete sentences like (iii) when conjoined with other true concrete sentences like (i) and (ii). In contrast, ' $1+1=3$ ' is concretely inadequate because it entails false concrete sentences like 'I had three apples' when conjoined with true concrete sentences like (i) and (ii).

A true mathematical sentence is a perfect inference-facilitator, whereas a false mathematical sentence is an imperfect inference-facilitator. To use an analogy, a true mathematical sentence acts like a system that takes true concrete sentences as an input and gives out only other true concrete sentences as an output. It never produces a false concrete sentence when given a true concrete sentence. In contrast, a false mathematical sentence acts like a system that takes true concrete sentences as an input but can give out a false concrete sentence as an output. This analogy, however, is not intended to justify inferentialism but to clarify inferentialism. 
Inferentialism asserts that a mathematical sentence is true if and only if it is concretely adequate, and that it is false if and only if it is concretely inadequate. For example, ' $1+1=2$ ' is true because it is concretely adequate, i.e., because when conjoined with true concrete sentences, it can issue only true concrete sentences. In contrast, ' $1+1=3$ ' is false because it is concretely inadequate, i.e., because when conjoined with true concrete sentences, it can issue a false concrete sentence. On the inferentialist account, to reiterate, mathematical truth coincides with concrete adequacy, and mathematical falsity coincides with concrete inadequacy. This semantic thesis about a mathematical sentence is also an important constituent of inferentialism.

What makes a mathematical sentence true or false? Inferentialists answer that it is the concrete world. A mathematical sentence is true or false, depending on whether its concrete consequences are true or false, and the concrete consequences are true or false in virtue of the way the concrete world is. In other words, the truth-value of a mathematical sentence is determined by the truth-values of its concrete consequences, and the truth-values of the concrete consequences are determined by the concrete world. So it is ultimately the concrete world, through a mode of correspondence, that renders a mathematical sentence true or false.

Do all mathematical sentences have truth-values? Inferentialists answer in the negative. A mathematical sentence does not have a truth-value unless it is used to deduce concrete sentences from other concrete sentences. In 1968, for example, the Italian physicist, Gabriele Veneziano (1942-present), was in search of mathematical equations that would explain the strong nuclear force that holds protons and neutrons together. He encountered a two-hundredyear-old mathematics book in which he found equations written down by the Swiss mathematician, Leonhard Euler (1707-1783). Euler devised the equations purely out of mathematical curiosity, and he did not use them to explain any phenomena. Two hundred years later, Veneziano first related them to the concrete world. On the inferentialist account, Euler's equations did not have truth-values before Veneziano used them to explain the strong force.

It should be clear from the preceding discussion on the truth-makers and the truthvalues of mathematical sentences that inferentialism denies the existence of the abstract world as well as rejects the realist semantic thesis that a mathematical sentence is true or false, depending on whether the abstract world is as it says it is. The abstract world simply does not figure in inferentialism. Thus, inferentialism includes the metaphysical thesis that the abstract world does not exist.

Inferentialism also contains the epistemological thesis that a mathematical sentence is confirmed and disconfirmed, just in case some of its concrete consequences are ascertained to be true and false, respectively. So it is subject to the tribunal of experience, just as a scientific theory is. Confirming a mathematical sentence does not require that we should confirm all of its concrete consequences. We only need to confirm some of its concrete consequences because ampliative inference is considered to be legitimate in the debate between inferentialists and realists.

A referee asks where mathematical sentences come from. My answer is that they come from experience and reason. Reason is the cognitive faculty that enables us to make inferences. Thanks to this cognitive faculty, I can infer that $1+1=2$, after experiencing that I had two apples as a result of receiving an apple from John and another apple from Jane. My acquisition of the mathematical sentence does not require that the abstract world should exist and cause the mathematical sentence in my mind. Even if the abstract world does not exist, we can acquire mathematical sentences. Also, a mathematical sentence can have concrete consequences, even if the abstract world does not exist. After all, concrete consequences are 
nothing but concrete sentences. We acquire concrete sentences from experience, as the foregoing example of the apples illustrates.

A referee suggests that I distinguish between inferentialism and instrumental nominalism (Rosen, 2001: 75; Ketland, 2011: 204). Instrumental nominalism is an antirealist response to the Quine-Putnam indispensability argument (Quine, 1948; 1980; 1992; Putnam, 1971; Resnik, 1997; Colyvan, 2001). The argument states that mathematical statements are indispensable components of our best scientific theories, that our best scientific theories are highly confirmed by observations, and hence that the mathematical statements are worthy of our beliefs. Instrumental nominalists retort that we can only believe that our best scientific theories nominalistically adequate, i.e., what they say about concreta are true. Thus, instrumental nominalists believe what our best scientific theories say about the concrete world, but do not believe what they say about the abstract world.

Let me point out one similarity and one difference between inferentialism and instrumental nominalism. The similarity is that both views make use of van Fraassen's notion of empirical adequacy in order to avoid mathematical realism. The difference is that while instrumental nominalism is designed to discriminate between trustworthy and untrustworthy claims of our best scientific theories, inferentialism is designed to illuminate the inferential role that a mathematical sentence plays with respect to concrete sentences. Recall that according to inferentialism, a true mathematical sentence is a perfect inference-facilitator, and a false mathematical sentence is an imperfect inference-facilitator.

In summation, inferentialism is composed of the functional thesis, the semantic thesis, the metaphysical thesis, and the epistemological thesis. These four theses are intricately connected with one another, forming a coherent whole, and this coherent set has several virtues, as will become clear in the following section.

\subsection{Virtues}

First, inferentialism explains why a mathematical sentence like ' $1+1=2$ ' was not refuted in the history of science. ' $1+1=2$ ' captures the deductive relationship between concrete sentences. Inferentialists would be happy to abandon ' $1+1=2$,' if a counterexample is offered, i.e., if a false concrete sentence is deducible from the conjunction of ' $1+1=2$ ' with true concrete sentences. No such counterexample was put forward in the history of science. Such a counterexample cannot even be conceived, given that a false concrete sentence cannot be deduced from the conjunction of ' $1+1=2$ ' with true concrete sentences. It is not surprising that ' $1+1=2$ ' was not discarded in the history of science.

Second, inferentialism secures the objectivity of mathematics. A mathematical sentence is true not because we think that it is true but because when conjoined with true concrete sentences, it can issue only true concrete consequences. The concrete sentences are true not because we think that they are true but because the concrete world is as they say it is, i.e., because they correspond to the concrete world. Their truth-values are determined by the concrete world. To take an example, the concrete sentence 'I had two apples' is true not because we think that it is true but because it corresponds to the concrete world. It follows that it is ultimately the concrete world that makes a mathematical sentence true, and hence that a mathematical sentence is true as objectively as a concrete sentence is true.

Third, inferentialism also explains why mathematics is successfully and widely used in empirical science. Mathematics is useful in empirical science because it shows which concrete sentences necessarily follow from which other concrete sentences. With the help of mathematics, scientists can make deductive inferences from the sentences describing the known aspect of the concrete world to the sentences describing the unknown aspect of the concrete world. Once they see the logical relationship between the concrete sentences, they 
can make true predictions about the unknown aspect of the concrete world. Thus, inferentialism presents a coherent picture or systematic narrative of how mathematics is a useful tool to investigate the concrete world.

Another referee asks the following questions. If a mathematical sentence shows which concrete sentences follow from which other concrete sentences, shouldn't it already have a truth-value in order to do that? How can its truth-value be determined by the concrete world?

Answers to these questions can be found in instrumentalists' insights. They claim that a scientific theory does not have a truth-value, and yet can show which observational sentences follow from which other observational sentences. Similarly, I claim that even if a mathematical sentence does not already have a truth-value, it can show which concrete sentences follow from which other concrete sentences. Also, on the inferentialist account, a mathematical sentence is true just in case it is concretely adequate, i.e., all of its concrete consequences are true. The concrete consequences are true or false, depending on the way the concrete world is. Therefore, their truth-values are determined by the concrete world.

Fourth, under the inferentialist framework, it is not mysterious how we acquire mathematical knowledge. We know that a mathematical sentence is true, only if we know that some of its concrete consequences are true. Thus, we can acquire mathematical knowledge via acquiring concrete knowledge. Acquiring concrete knowledge is not considered to be problematic in the debate over the ontological status of mathematical objects. All rivaling participants in the debate take it to be uncontroversial that we can have concrete knowledge. To question our ability to acquire concrete knowledge is to at once question the very starting point of the debate.

The referee suggests that I should explain what I mean by 'mathematical knowledge.' Didn't Euler have mathematical knowledge before his equations were used by Beneziano in 1968 ?

Set aside the Gettier problem, and suppose for the sake of argument that Plato is right that knowledge is justified true belief. I believe that $1+1=2$. It is true that $1+1=2$ in that all of its concrete consequences are true. I am justified in believing that $1+1=2$ in that $I$ can confirm some of its concrete consequences. Therefore, I have the justified true belief that $1+1=2$. Euler did not have the mathematical knowledge concerning his equations, for the equations did not even have truth-values, and he did not have observational evidence for them.

\subsection{Objections and Replies}

Let me now turn to possible objections to inferentialism. Objectors might say that there are mathematical sentences asserting how mathematical objects are related to concrete objects. They are such sentences as 'The number of tigers in this zoo is 5' and 'The distance, in meters, is 10.' Let me call such sentences connecting sentences. The idea is that they seem to connect mathematical objects with concrete objects. They imply that mathematical objects exist, and that mathematical objects are related to concrete objects in a certain manner. Thus, they undermine inferentialism, which denies the existence of mathematical objects.

How do inferentialists respond to the preceding objection? We should not be misled by the linguistic structure of a connecting sentence, just as we should not be misled by the linguistic structure of a bridge principle. A scientific theory is composed of internal and bridge principles, as Carl G. Hempel (1966: 72-75) observes. An internal principle is a claim about how theoretical entities behave. It is couched only in theoretical terms. By contrast, a bridge principle is a claim about how the behavior of the theoretical entities is related to observational entities. It is couched in both theoretical terms and observational terms. For example, the caloric theory of heat includes the bridge principle that temperature is proportional to the amount of caloric fluids. Note that the linguistic structure of the bridge 
principle indicates that caloric fluids exist when they do not. Instrumentalists argue that theoretical terms like 'caloric fluids' are merely useful instruments for organizing our thoughts about observables, although they appear to refer to theoretical entities when they are used in sentences. Analogously, inferentialists argue that numerals like ' 5 ' and ' 10 ' are merely useful instruments to accurately represent the concrete states of affairs, although they appear to refer to abstract objects when they are used in sentences.

Let me move to another possible objection to inferentialism. I argued that ' $1+1=2$ ' can deductively organize concrete sentences, but I provided only one example of a group of concrete sentences deductively organized by the mathematical sentence. How do we know whether a certain group of concrete sentences are deductively organized by a mathematical sentence or not? Inferentialism is an incomplete theory, critics might argue, until I specify all the concrete sentences the mathematical sentence deductively organizes, or until I provide an account of how we go about determining whether or not a group of concrete sentences are deductively organized by a mathematical sentence.

In my view, the foregoing criticism is misguided. Recall that van Fraassen claims that a scientific theory is empirically adequate if and only if all of its observational consequences are true. He has never enumerated all the empirical consequences of a scientific theory to flesh out his position. Even so, his position has never been regarded as being incomplete in the philosophy of science literature.

Let me turn to yet another possible objection to inferentialism. Objectors might say that inferentialism cannot handle the validity of arguments with pure mathematical premises and conclusions. Consider, for example, the following argument:

$$
\begin{gathered}
2 x+3 y=13 \\
3 x-y=3 \\
\therefore x=2 \text {, and } y=3
\end{gathered}
$$

The premises and the conclusion of this argument are all pure mathematical sentences in that they are not applied to the concrete world. Even if they are independent of the concrete world, the argument is deductively valid. But why is it deductively valid? How can inferentialism make sense of deductive validity?

My answer to this question is that it is wrong to think that inferentialism does not allow for mathematical sentences to form a deductively valid or sound argument. To use an analogy, van Fraassen believes that thermodynamics and statistical mechanics are merely empirically adequate. Even so, he can believe that thermodynamics is deducible from statistical mechanics. It is not the case that since he believes that they are merely empirically adequate, he cannot believe that thermodynamics is deducible from statistical mechanics. Likewise, inferentialists believe that true mathematical sentences are merely concretely adequate. Even so, they can believe that a mathematical sentence is deducible from other mathematical sentences. It is not the case that since they believe that mathematical sentences are merely concretely adequate, they cannot believe that a mathematical sentence is deducible from other mathematical sentences.

\section{Disadvantages of Realism}

Realism has three disadvantages over inferentialism. First, realism claims that the abstract world exists. It is inhabited by abstract entities, such as mathematical objects and propositions. They are aspatial and atemporal entities. Hence, they are causally inert. They can interact neither with one another nor with concrete entities, such as electrons, black holes, and human beings. According to realism, ' $1+1=2$ ' is true not because we think that it is true 
but because the abstract world is as it says it is. Thus, realism secures the objectivity of mathematics by postulating the existence of the abstract world. In contrast, as I have outlined in Section 2, inferentialism does it without positing the existence of abstract world. Therefore, the principle of economy dictates us to choose inferentialism over realism.

Second, under the realist framework, it is mysterious how we can acquire mathematical knowledge (Benacerraf, 1973; Balaguer, 2014). As stated earlier, abstract entities are causally inert, so they cannot interact with concrete entities, including human beings. We usually acquire knowledge about a concrete object by interacting with it. For example, we interact with a cat, and as a result, we come to know about it. It is impossible, however, for us to interact with a triangle. Consequently, realists owe us an account of how mathematical knowledge is possible. In contrast, inferentialists already have an uncontroversial account of how mathematical knowledge is possible, as we have seen in Section 2.

Third, under the realist framework, it remains unexplained why mathematics is useful in empirical science. A mathematical sentence is a description of the abstract world, not of the concrete world. It is not clear, however, how the description of the abstract world can be useful in investigating the concrete world, given that the abstract world is aspatial and atemporal while the concrete world is both spatial and temporal. Suppose that we want to investigate the abstract world. We want to know what the abstract world looks like, what its construct is like, what type of objects inhabits such a world, what properties the inhabitants have, and so forth. Can concrete sentences be useful in the new discipline? Consider concrete sentences like 'All human beings are mortal.' Such a sentence is about concrete objects that change over time. Hence, it is useless in organizing our thoughts about abstracta that do not change over time. Similarly, a mathematical sentence, if it is a description of the abstract world, should be useless in empirical science. But it is obvious that mathematical sentences are useful in empirical science. This obvious fact is an anomaly to realism.

In response, realists might embrace the inferentialist functional thesis that a mathematical sentence can perform the function of facilitating deductive inferences from concrete sentences to other concrete sentences, while adhering to the realist semantic thesis that a mathematical sentence performs the function of describing the abstract world. They can then explain why mathematics is useful in empirical science without abandoning the realist semantic thesis.

This response, however, is problematic. The inferentialist functional thesis and the realist semantic thesis conflict with each other, as we have seen in this section. It is implausible that a description of the abstract world can be useful in organizing concrete sentences. Furthermore, once realists embrace the inferentialist functional thesis, it is otiose to say that the abstract world exists, and that a mathematical sentence is true or false in virtue of the way the abstract world is. The realist metaphysical thesis and semantic thesis are superfluous because only the inferentialist functional thesis is necessary to explain the successful use of mathematics in empirical science and to secure the objectivity of mathematics. Again, Ockham's razor militates against realism.

\section{Disadvantages of Fictionalism}

Fictionalism in philosophy of mathematics is the view that "(a) our mathematical sentences and theories do purport to be about abstract mathematical objects, as platonism ${ }^{1}$ suggests, but (b) there are no such things as abstract objects, and so (c) our mathematical theories are not true" (Balaguer, 2013). Keep in mind that fictionalists accept the realist semantic thesis that a mathematical sentence is a description of the abstract world, but reject the realist

\footnotetext{
${ }^{1}$ Mathematical realism is also called platonism in philosophy of mathematics. It differs from Platonism, the philosophy of Plato.
} 
metaphysical thesis that mathematical objects exist in the abstract world, and hence accept the corollary that all mathematical sentences are false. I strictly follow Balaguer's formulation of fictionalism in this paper.

Fictionalism, as defined by Balaguer above, is similar to John L. Mackie's (1977) error theory in metaethics. The error theory holds that a moral sentence is not an expression of emotion but a description of the world. However, all moral sentences are false because moral properties and facts do not inhabit the world. Thus, error theorists accept the moral realist semantic thesis that a moral sentence has a truth-value, but reject the moral realist metaphysical thesis that there are moral properties and facts. Both error theorists and fictionalists accept their realist counterparts' semantic theses but reject their realist counterparts' metaphysical theses.

Let's now turn to the comparison between fictionalism and inferentialism. There are an important similarity and an important difference between them. The similarity is that both views reject the realist metaphysical thesis that mathematical objects are real. The difference is that fictionalism affirms, while inferentialism denies, that a mathematical sentence purports to be about the abstract world, and that all mathematical sentences are false. Recall that, according to inferentialism, the abstract world does not exist, and a mathematical sentence is true or false, depending on whether all of its concrete consequences are true or not. Hence, some mathematical sentences, such as ' $1+1=2$,' are true, and other mathematical sentences, such as ' $1+1=3$,' are false.

Fictionalism suffers from three disadvantages that inferentialism does not. First, fictionalism cannot explain why mathematics is useful in empirical science. Fictionalism claims that mathematical sentences are descriptions of the abstract world. As noted in Section 3 , it is not clear how a description of the aspatial and atemporal world can be useful in empirical science which aims to explain and predict events that occur in space and time. Of course, a false scientific theory can be successful. For example, the Ptolemaic theory was successful in predicting the motions of celestial bodies in the past. There is, however, a fundamental difference between a false scientific theory and a false mathematical sentence. A false scientific theory is a description of the concrete world, whereas the false mathematical sentence is a description of the abstract world.

Second, fictionalism attributes falsities to mathematical beliefs, whereas inferentialism does not. Ordinary folk believe, for example, that ' $1+1=2$ ' is true. Fictionalists claim that their belief is false, whereas inferentialists claim that it is true. The principle of charity requires that we should not ceteris paribus attribute false beliefs to others. Both inferentialism and fictionalism strive for avoiding the realist metaphysical thesis that mathematical objects are abstract entities. In the process of achieving that aim, inferentialism complies with, while fictionalism violates, the principle of charity.

In addition, it is implausible that ordinary folk really have the intention that their mathematical sentences are descriptions of the abstract world. Ordinary folk are not even aware of the implications of what it is for an object to exist outside of space and time. For example, even God cannot do anything in the abstract world. He cannot even move his finger because the motion of his finger requires the flow of time and because time does not pass in the abstract world (Park, 2017: 164). Also, an atemporal and aspatial object is not visualizable. It is psychologically impossible to form the image of an object that does not occupy space and time. As Stephen Kosslyn (1980: 1) observes, however, ordinary people often think in terms of pictures. When asked which is larger, a tennis ball or a volleyball, they form the images of a tennis ball and a volleyball in their minds, and then compare them. These considerations support the view that ordinary folk do not believe that their 
mathematical sentences are descriptions of the abstract world. Only highly trained people like philosophers have the intention to describe the abstract world with mathematical sentences.

Third, it is not clear how fictionalism can secure the objectivity of mathematics. Is there an objective basis for choosing ' $1+1=2$ ' over ' $1+1=3$ '? If there is, what is it? Fictionalists cannot say that we ought to choose the former over the latter because the former is true and the latter is false, for they claim that all mathematical sentences are false. What then is the difference between ' $1+1=2$ ' and ' $1+1=3$ '? Balaguer $(2001 ; 2009 ; 2013)$ answers this question from a fictionalist point of view. In my view, however, his answer is not convincing.

Balaguer suggests that ' $1+1=2$ ' is fictionalistically correct, whereas ' $1+1=3$ ' is fictionalistically incorrect. What does it mean to say that a mathematical sentence is fictionalistically correct?

..a mathematical sentence is fictionalistically correct if and only if it would have been true if there had actually existed abstract mathematical objects of the kinds that platonists have in mind. (Balaguer, 2013)

Thus, ' $1+1=2$ ' is fictionalistically correct because if the abstract world exists, then it would be true, and ' $1+1=3$ ' is fictionalistically incorrect because if the abstract world exists, then it would be false. The abstract world does not exist. But if it does, then it would make ' $1+1=2$ ' true and ' $1+1=3$ ' false. Thus, a counterfactual making use of the abstract world can be used to determine whether a mathematical sentence is fictionalistically correct or incorrect.

Balaguer's proposal, however, can be reduced to absurdity. If his proposal is correct, then it should also be legitimate to say that ' $1+1=3$ ' and 'The Earth is flat' are fictionalistically correct because if another abstract world in which $1+1=3$ exists, then ' $1+1=3$ ' would be true, and if another concrete world in which the Earth is flat exists, then 'The Earth is flat' would be true. Neither that abstract world nor that concrete world exists. But if they did, then ' $1+1=3$ ' and 'The Earth is flat' would be true. It is not clear on what grounds Balaguer could say that (1) is legitimate, but (2) and (3) are illegitimate:

(1) ' $1+1=2$ ' is fictionalistically correct because if the abstract world exists, then it would be true.

(2) ' $1+1=3$ ' is fictionalistically correct because if another abstract world in which $1+1=3$ exists, then it would be true.

(3) 'The Earth is flat' is fictionalistically correct because if another concrete world in which the Earth is flat exists, then it would be true.

Thus, ' $1+1=2$,' ' $1+1=3$,' and 'The Earth is flat' are all on the same boat. Any mathematical sentence or any concrete sentence could be made fictionalistically correct simply by formulating a relevant counterfactual.

The preceding discussion is intended to show that Balaguer's distinction between fictionalistically correct and incorrect sentences is untenable, and that Balaguer's attempt to secure the objectivity of mathematics fails. The failure of his proposal stems from the fact that it invokes the abstract world rather than the concrete world to establish the objectivity of mathematics. Given that the abstract world is not the only world available for the formulation of a relevant counterfactual, it is not clear which worlds we should choose and which worlds we should not choose to formulate a relevant counterfactual. So it is a mistake in the first place to try to ground the objectivity of mathematics on a counterfactual invoking the abstract world. 
In my view, the objectivity of mathematics should be grounded on the concrete world rather than on the abstract world or on a counterfactual making use of the abstract world. As we have seen in Section 2, inferentialism claims that the concrete world contributes to the objectivity of mathematics. Any philosophical account of the objectivity of mathematics that does not recognize the contribution of the concrete world is missing something important.

How would fictionalists respond to my preceding discussion? They might object that I mischaracterized fictionalism. Fictionalism does not claim that all mathematical sentences are false. It rather claims that some mathematical sentences are false, and that other mathematical sentences are true. Fictionalists are well aware that the negation of a mathematical sentence is also a mathematical sentence, and that it is impossible for both of them to be false. For example, it is impossible that both 'The sum of the three angles of a triangle is 180 degrees' and 'The sum of the three angles of a triangle is not 180 degrees' are false. If one is false, then the other must be true, and vice versa. So fictionalism allows for true mathematical sentences.

In my view, however, it is wrong to think that if a sentence is false, then its negation must be true. It is possible for both of them to be false. Consider the sentences, 'The present king of France is bald' and 'The present king of France is not bald.' One of them is the negation of the other. But both of them are false because they are about an object that does not exist. Similarly, it is wrong to think that either a mathematical sentence or its negation is true. On the fictionalist account, both of them are false because they are about an object that does not exist.

Moreover, I am following Balaguer on the interpretational issue of whether fictionalism claims or not that all mathematical sentences are false. Recall that in the beginning of this section I quoted Balaguer's definition of fictionalism according to which "our mathematical theories are not true" (Balaguer, 2013). If there are fictionalists in the literature who claim that some mathematical sentences are true, my preceding argument from the principle of charity does not apply to them. I leave, however, to those fictionalists the thorny issue of how to cash out the objectivity of mathematics and the difference between the truth and the falsehood of a mathematical sentence within the fictionalist framework.

\section{Conclusion}

Inferentialism has some virtues. It explains why a mathematical sentence like ' $1+1=2$ ' was not and could not be refuted in the history of science. It secures the objectivity of mathematics and accounts for the successful use of mathematics in empirical science. It presents an uncontroversial account of how we acquire mathematical knowledge. It has these virtues, and yet it does not postulate the existence of the abstract world. So it is a good philosophical theory of mathematics from the point of view of Ockham's razor.

Both realism and fictionalism have disadvantages over inferentialism. Realism secures the objectivity of mathematics at the cost of positing the existence of the abstract world. It faces the difficult task of explaining how we can acquire mathematical knowledge and why mathematics is useful in empirical science. Fictionalism does not fare better than realism. It also faces the daunting task of explaining why mathematics is useful in empirical science. It is saddled with an implausible assumption that ordinary folk have a clear conception of the abstract world. Finally, it jeopardizes the objectivity of mathematics.

To conclude, there are fewer obstacles on the road to inferentialism than on the roads to realism and fictionalism.

\section{References}


Baker, Alan (2005). "Are There Genuine Mathematical Explanations of Physical Phenomena?", Mind 114 (454): 223-237.

(2009). "Mathematical Explanation in Science", British Journal for the Philosophy of Science 60 (3): 611-633.

(2012). “Science-Driven Mathematical Explanation”, Mind 121 (482): 243-267.

Balaguer, Mark (1996). "Towards a Nominalization of Quantum Mechanics", Mind 105 (418): 209-206.

Press.

(1998). Platonism and Anti-Platonism in Mathematics. Oxford: Oxford University (2001). "A Theory of Mathematical Correctness and Mathematical Truth", Pacific Philosophical Quarterly 82 (2): 87-114.

(2009). "Fictionalism, Theft, and the Story of Mathematics", Philosophia Mathematica 17 (2): 131-162.

(2013). "Fictionalism in the Philosophy of Mathematics", The Stanford Encyclopedia of Philosophy. Edward N. Zalta (ed.). URL = <http://plato.stanford.edu/archives/fall2013/entries/fictionalism-mathamatics/>

(2014). "Platonism in Metaphysics", The Stanford Encyclopedia of Philosophy. Edward N. Zalta (ed.), URL = <http://plato.stanford.edu/archives/spr2014/entries/platonism/>.

Benacerraf, Paul (1973). "Mathematical Truth”, The Journal of Philosophy 70 (19): 661-679.

Colyvan, Mark (2001). The Indispensability of Mathematics. New York: Oxford University Press.

Downing, Lisa (2013). "George Berkeley", The Stanford Encyclopedia of Philosophy. Edward N. Zalta (eds.). URL = <http://plato.stanford.edu/archives/spr2013/entries/berkeley/>.

Duhem, Pierre (1905). The Aim and Structure of Physical Theories. (Phillip Wiener, Trans.) Princeton, NJ: Princeton University Press (1954).

Hempel, Carl G. (1966). Philosophy of Natural Science. Englewood Cliffs, NJ: Prentice-Hall.

Ketland, Jeffrey (2011). "Nominalistic Adequacy", Proceedings of the Aristotelian Society 111(2pt2): 201-217.

Kosslyn, Stephen M. (1980). Image and Mind. Cambridge: Harvard University Press.

Kuhn, Thomas S. (1957). The Copernican Revolution: Planetary Astronomy in the Development of Western Thought. Cambridge: Harvard University Press.

Leng, Mary (2005a). "Revolutionary Fictionalism: A Call to Arms", Philosophia Mathematica, 13 (3): 277-293. 
(2005b). "Mathematical Explanation”, In Mathematical Reasoning and Heuristics. C. Cellucci and D. Gillies (eds.), London: King's College Publication.

(2010). Mathematics and Reality. Oxford: Oxford University Press.

Mackie, John L. (1977). Ethics: Inventing Right and Wrong. Harmondsworth: Penguin.

Park, Seungbae (2017). "The Problems of Divine Location and Age", European Journal of Science and Theology 13 (2): 41-53.

Putnam, Hilary (1971). Philosophy of Logic. New York: Harper and Row.

Quine, Willard V. O. (1948) “On What There Is”, Review of Metaphysics 2 (5): 21-38.

Press.

(1980). From a Logical Point of View. $2^{\text {nd }}$ ed., Cambridge, MA: Harvard University

--------- (1992). Pursuit of Truth. Cambridge MA: Harvard University Press.

Resnik, Michael (1997). Mathematics as a Science of Patterns. Oxford: Clarendon Press.

Rosen, Gideon (2001). "Nominalism, Naturalism, Epistemic Relativism”, Noûs 35 (s15): 6091.

van Fraassen, Bas (1980). The Scientific Image. Oxford: Oxford University Press.

(1985). "Empiricism in the Philosophy of Science", In Images of Science. Paul M. Churchland and Cliff A. Hooker (eds.), Chicago: University of Chicago Press. 